APS

physics

This is the accepted manuscript made available via CHORUS. The article has been published as:

\title{
Logarithmic Temperature Profiles in Turbulent Rayleigh- Bénard Convection
}

Guenter Ahlers, Eberhard Bodenschatz, Denis Funfschilling, Siegfried Grossmann, Xiaozhou He, Detlef Lohse, Richard J. A. M. Stevens, and Roberto Verzicco

Phys. Rev. Lett. 109, 114501 - Published 14 September 2012 DOI: 10.1103/PhysRevLett.109.114501 


\title{
Logarithmic temperature profiles in turbulent Rayleigh-Bénard convection
}

\author{
Guenter Ahlers ${ }^{1,2}$, Eberhard Bodenschatz ${ }^{2,3,4}$, Denis Funfschilling ${ }^{5}$, Siegfried Grossmann ${ }^{6}$, \\ Xiaozhou $\mathrm{He}^{2}$, Detlef Lohse ${ }^{7}$, Richard J.A.M. Stevens ${ }^{7}$, and Roberto Verzicco ${ }^{7,8}$ \\ ${ }^{1}$ Department of Physics, University of California, Santa Barbara, CA 93106, USA \\ ${ }^{2}$ Max Planck Institute for Dynamics and Self-Organization, D-37073 Goettingen, Germany \\ ${ }^{3}$ Institute for Nonlinear Dynamics, University of Göttingen, D-37073 Göttingen, Germany \\ ${ }^{4}$ Laboratory of Atomic and Solid-State Physics and Sibley School of Mechanical \\ and Aerospace Engineering, Cornell University, Ithaca, New York 14853 \\ ${ }^{5}$ LSGC CNRS - GROUPE ENSIC, BP 451, 54001 Nancy Cedex, France \\ ${ }^{6}$ Fachbereich Physik der Philipps-Universität, Renthof 6, D-35032 Marburg, Germany \\ ${ }^{7}$ Department of Science and Technology and J.M. Burgers Center for Fluid Dynamics, \\ University of Twente, P.O Box 217, 7500 AE Enschede, The Netherlands and \\ ${ }^{8}$ Dept. of Mech. Eng., Universita di Roma Tor Vergata, Via del Politecnico 1, 00133, Roma.
}

(Dated: June 26, 2012)

\begin{abstract}
We report results for the temperature profiles of turbulent Rayleigh-Bénard convection (RBC) in the interior of a cylindrical sample of aspect ratio $\Gamma \equiv D / L=0.50$ ( $D$ and $L$ are the diameter and height respectively). Both in the classical and in the ultimate state of RBC we find that the temperature varies as $A \times \ln (z / L)+B$ where $z$ is the distance from the bottom or top plate. In the classical state the coefficient $A$ decreases in the radial direction as the distance from the side wall increases. For the ultimate state the radial dependence of $A$ has not yet been determined. These findings are based on experimental measurements over the Rayleigh-number range $4 \times 10^{12} \lesssim R a \lesssim$ $10^{15}$ for a Prandtl number $\operatorname{Pr} \simeq 0.8$ and on direct numerical simulation at $R a=2 \times 10^{12}, 2 \times 10^{11}$, and $2 \times 10^{10}$, all for $\operatorname{Pr}=0.7$.
\end{abstract}

PACS numbers: 47.27.te,47.32.Ef,47.20.Bp,47.27.ek

Turbulent convection of a fluid contained between two horizontal plates separated by a distance $L$ and heated from below (Rayleigh-Bénard convection or RBC) [1-3] is a system in fluid mechanics with many features that are of fundamental interest. It is also a phenomenon with numerous astrophysical [4-6], geophysical [7-12], and technological $[13,14]$ applications. Nonetheless some of its properties remain incompletely explored and understood.

In turbulent RBC a "classical" state exists below a transition range to an "ultimate" state; for the fluid used by us the transition range extends from $\mathrm{Ra}_{1}^{*} \simeq 2 \times 10^{13}$ to $\mathrm{Ra}_{2}^{*} \simeq 5 \times 10^{14}$ [15] ( $\mathrm{Ra}$ is a dimensionless measure of the applied temperature difference). For the classical state it is known from experiment (see, for instance, [1623]) that approximately half of the applied temperature difference $\Delta T \equiv T_{b}-T_{t}\left(T_{b}\right.$ and $T_{t}$ are the temperatures at the bottom and top of the sample respectively) is sustained by two thin thermal boundary layers (BLs), one just below the top and the other just above the bottom plate. These BLs are laminar, albeit fluctuating [24-26]. The entire interior of the sample, known as the "bulk", is then approximately isothermal in the time average, but it also undergoes vigorous local temperature fluctuations [27]. For the ultimate state it was predicted [28] that the BLs are turbulent as well, due to the shear that is applied by vigorous fluctuations and possibly by a large-scale circulation (LSC) in the bulk. These turbulent BLs are expected to extend throughout the sample and to produce a temperature field (beyond a very thin thermal sublayer adjacent to the plates) that varies logarithmically with the distance from the plates.

For both the classical and the ultimate state we found from experiment that, beyond a thin BL or thermal sublayer (which was unresolved by experiment) the temperature $T(z)$ and its root-mean-square (rms) fluctuation $\sigma(z)$ vary logarithmically as a function of the distance $z$ from the bottom plate. For the classical state these results were confirmed and extended by direct numerical simulation (DNS). These findings agree with the logarithmic dependence predicted for the ultimate state above $\mathrm{Ra}_{2}^{*}$ [28], but to our knowledge there is no theory at present that predicts a logarithmic temperature profile in the bulk for $\mathrm{Ra}<\mathrm{Ra}_{1}^{*}$. We believe that the discovery of logarithmic profiles is an important step towards developing a more fundamental understanding of the bulk.

The apparatus $[29,30]$ and the numerical method $[31,32]$ were described before. In the experiment we used sulfur hexafluoride at pressures up to 19 bars and at $T_{m} \simeq 21^{\circ} \mathrm{C}$ as the fluid. The Prandtl number $\operatorname{Pr}$ was $0.79(0.86)$ near $\mathrm{Ra}=4 \times 10^{12}\left(10^{15}\right)$. The sample was tilted slightly, with its axis at an angle of $14 \mathrm{mrad}$ relative to gravity. This assured that any remnants of a LSC that survive at these large Ra [15] would, on average, choose a preferred azimuthal up-flow and down-flow orientation (see, for instance, $[33,34]$ ). The tilting had no other effects on our results (for details, see [30]). Two sets of thermistors were installed for the temperatureprofile measurements. One was located at what would be the preferred down-flow orientation at lower $R a$, and the other was removed from the first in the azimuthal 
direction by an angle $\pi$. Each set consisted of eight thermometers which were located in the fluid $1.0 \pm 0.1 \mathrm{~cm}$ from the side wall. The eight thermistors were located at $z=4.0,6.1,8.1,12.1,16.1,32.2,64.2$, and $110.5 \mathrm{~cm}$, with an uncertainty of the vertical position of $0.1 \mathrm{~cm}$.

We present experimental measurements over the range $4 \times 10^{12} \lesssim \mathrm{Ra} \lesssim 10^{15}$. They are for a single $(R-r) / L=$ $0.0045(R=D / 2$ and $r$ is the radial coordinate). This location is well inside the bulk; the DNS data at $\mathrm{Ra}=$ $2 \times 10^{12}$ showed that the viscous BL only extends to $(R-r) / L \approx 0.0008$. We also analyze DNS data for $\operatorname{Pr}=$ 0.7 and $\mathrm{Ra}=2 \times 10^{10}, 2 \times 10^{11}$, and $2 \times 10^{12}[31,32]$ for a cylindrical sample of aspect ratio $\Gamma \equiv D / L=0.50(D$ is the diameter). They cover the entire radial position range $0 \lesssim(R-r) / L \lesssim 0.25$. Both experiment and DNS show that, through much of the bulk, the dimensionless time-averaged temperature $\Theta(z) \equiv\left[\langle T(z)\rangle-T_{m}\right] / \Delta T$ (we denote the time average by $\langle\ldots\rangle$ and $\left.T_{m} \equiv\left(T_{b}+T_{t}\right) / 2\right)$ can be represented well by

$$
\Theta(z)=A \times \ln (z / L)+B
$$

From the experiment we also find that the rms temperature fluctuations $\sigma(z) \equiv\left\langle[T(z)-\langle T(z)\rangle]^{2}\right\rangle^{1 / 2} / \Delta T$ are consistent with a logarithmic dependence on $z$, and represent them by

$$
\sigma(z)=C \times \ln (z / L)+D .
$$

The DNS data show that the amplitude $A(r)$ in the classical state is largest near the side wall and decreases as the distance $R-r$ from the wall increases.

Typical data sets for $\Theta$, each based on the sixteen time-averaged temperatures, are shown in Fig. 1(a) as a function of $z / L$ on a logarithmic scale. The stars (blue online, shown only for $\mathrm{Ra}=1.08 \times 10^{15}$ ) are results at the preferred down-flow and up-flow orientation of the LSC. They reveal a small difference at the two locations which is typical in size of all other measurements. We attribute this difference to the influence of remnants of the LSC on the temperature profiles [35]. Henceforth we consider only the average at each vertical position of the two data sets, as shown by the solid symbols in the figure. The lower (upper) two data sets are for the ultimate (classical) state. Except at the largest $z / L$, the data fall on straight lines and thus are represented well by Eq. 1. In the ultimate state the logarithmic dependence is followed within the uncertainty of the data for more than a decade of $z / L$, from $z / L=0.018$ to $z / L=0.3$, corresponding to a physical length up to $0.64 \mathrm{~m}$. A significant deviation is seen only at the largest $z / L=0.5$, i.e. at the sample center. For the classical state measurable deviations from the logarithmic form occur already at $z / L=0.3$. Note that these deviations are similar to what is known for the logarithmic profiles in pipe flow, which also do not extend right to the center of the pipe.

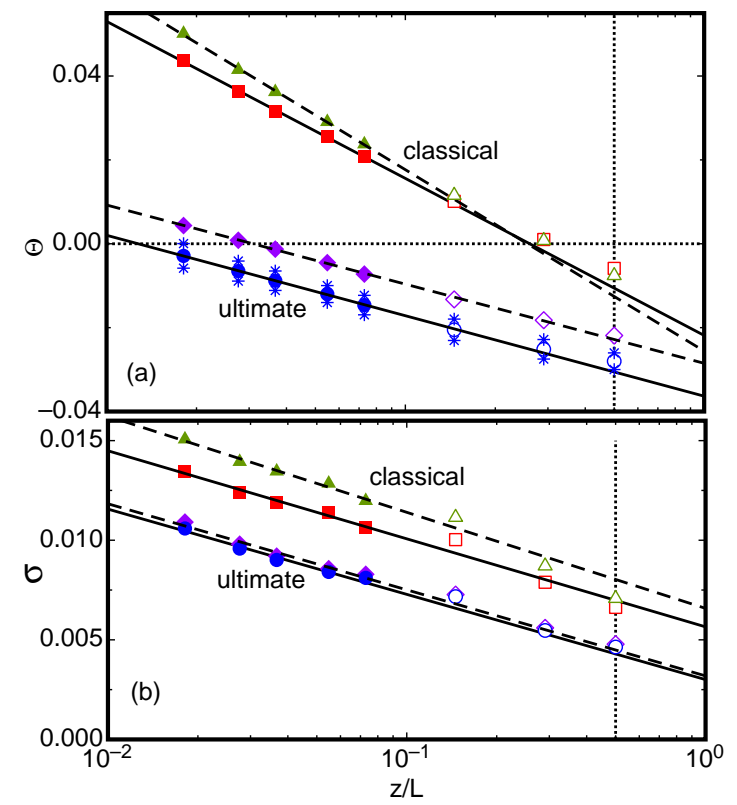

FIG. 1. Results at the radial location $(R-r) / L=0.0045$. (a): $\Theta(z) \equiv\left[\langle T(z)\rangle-T_{m}\right] /\left(T_{b}-T_{t}\right)$ and (b): $\sigma(z)=\langle[T(z)-$ $\left.\langle T(z)\rangle]^{2}\right\rangle^{1 / 2} /\left(T_{b}-T_{t}\right)$ as a function of vertical position $z / L$. Vertical dotted line: sample center at $z / L=0.5$. The data are for $\mathrm{Ra}=4.9 \times 10^{12}$ (triangles, green online), $1.18 \times 10^{13}$ (squares, red online), $7.9 \times 10^{14}$ (diamonds, purple online), and $1.08 \times 10^{15}$ (circles, blue online). Diamonds and circles in (b) are very close to each other. Stars are results at the preferred down-flow and up-flow orientation of the LSC for $\mathrm{Ra}=1.08 \times 10^{15}$. All other symbols are averages of the two locations. The solid symbols $(z / L \lesssim 0.08)$ were used for the fits of Eq. 1 or 2 to the data. The lines are those fits.

In a sample that conforms perfectly to the Boussinesq approximation we would expect another logarithmic dependence emanating from the top plate to meet the data shown in the figure at $T_{m}$ (i.e. at $\Theta=0$ ) and $z / L=1 / 2$. However, in the experiment we find that $\Theta(z / L=1 / 2)<0$, albeit only by $0.006(0.028)$ for $R a \simeq 10^{13}\left(10^{15}\right)$. Results for $\Phi \equiv\left(T_{c}-T_{m}\right) / \Delta T$ are given in Fig. 2(e). We do not know the reason for this offset. However, it will necessarily lead to a small departure from the logarithmic dependence because the two branches, one coming from the bottom and the other from the top plate, must have a continuous derivative at $z / L=1 / 2$ where they meet. For a quantitative analysis we therefore fit Eq. 1 only to the five points with $z / L \lesssim 0.08$. The resulting functions are shown as the lines in the figure, and the parameters $A$ and $B$ are given in Fig. 2(a) and (b). The offset $\Phi<0$ also shifts the constant $B$ in Eq. 1 . The corrected parameter $B-\Phi$ is shown in Fig. 2(b) as open circles. Although $B$ and $\Phi$ varied strongly in the ultimate state, $A$ and $B-\Phi$ are essentially constant above $\mathrm{Ra}_{2}^{*}$.

The rms temperature fluctuations $\sigma$ are shown in Fig. 1b. In analogy to recent measurements for turbulent 


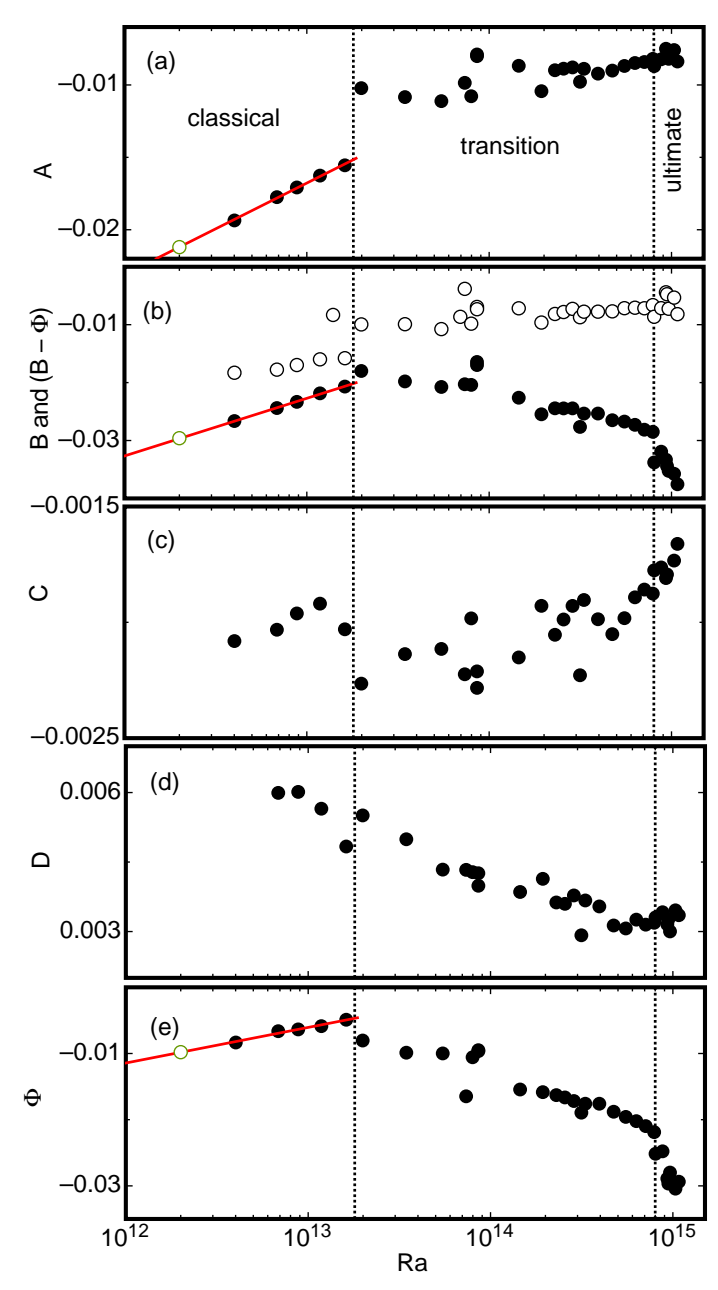

FIG. 2. The parameters $A, B, C$, and $D$ obtained by fitting Eqs. 1 and 2 to the experimental temperature and fluctuation profiles, and the deviation $\phi \equiv\left(T_{c}-T_{m}\right) / \Delta T$ of the temperature $T_{c}=T(z / L=1 / 2)$ from the mean temperature $T_{m}$, all as a function of Ra. The open circles in (b) represent $B-\Phi$. All data are for a radial position $(R-r) / L=0.0045$. The vertical dotted lines indicate the locations of $\mathrm{Ra}_{1}^{*}$ and $\mathrm{Ra}_{2}^{*}$. The solid lines (red online) are fits of the function $A=a * \log _{10}(\mathrm{Ra})+b$ to the data with $\mathrm{Ra}<\mathrm{Ra}_{1}^{*}$. The extrapolation to $\mathrm{Ra}=2 \times 10^{12}$ (open circles, green online) yielded $A=-0.0212, B=-0.0296$, and $\Phi=-0.0098$.

pipe flow [36], these fluctuations also follow a logarithmic form. Also in this case the relevant equation (Eq. 2) was fitted to the data only for $z / L \lesssim 0.08$ to determine $C$ and $D$, which are given in Fig. 2(c) and (d).

As discussed above, there is a range of Ra which extends from $\mathrm{Ra}_{1}^{*}$ to $\mathrm{Ra}_{2}^{*}$ (the vertical dotted lines in the figure) over which the transition from the classical to the ultimate state takes place [15]. The locations of $\mathrm{Ra}_{1}^{*}$ and $\mathrm{Ra}_{2}^{*}$ are particularly noticeable in the data for $B$ and $\Phi$. In the transition region the parameters scatter more than above or below it because the state assumed by the system can vary from one experimental point to another.

In Fig. 3 we show results for $\Theta(z)$ from DNS [31, 32].

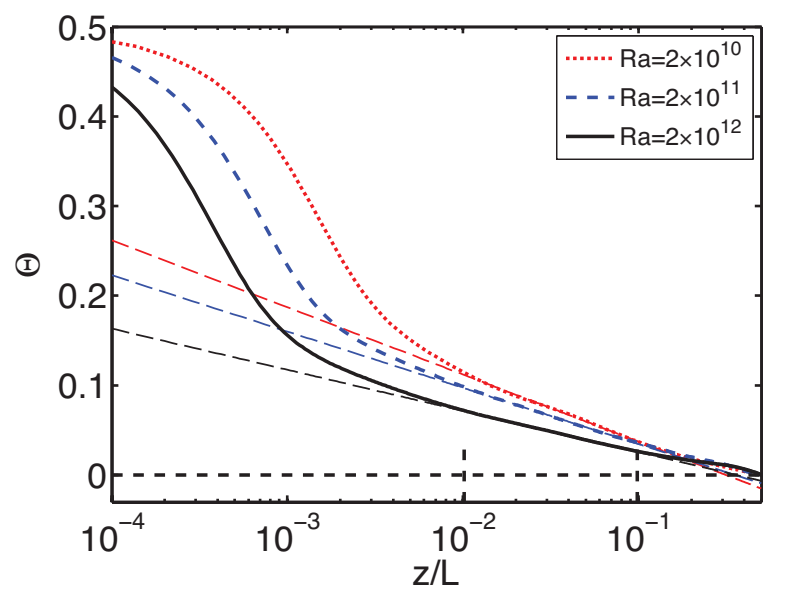

FIG. 3. $\Theta(z)$ as a function of $z / L$ at a radial position $(R-$ $r) / L=0.0045$ from DNS for three values of $\mathrm{Ra}, \operatorname{Pr}=0.7$, and $\Gamma=1 / 2$. Averages of the profiles measured as a function of distance from the bottom and the top plate are shown. The thin dashed straight lines are fits of Eq. 1 over the range $0.01 \leq z / L \leq 0.1$ to the data (the fitting interval is indicated by two short dashed vertical lines).

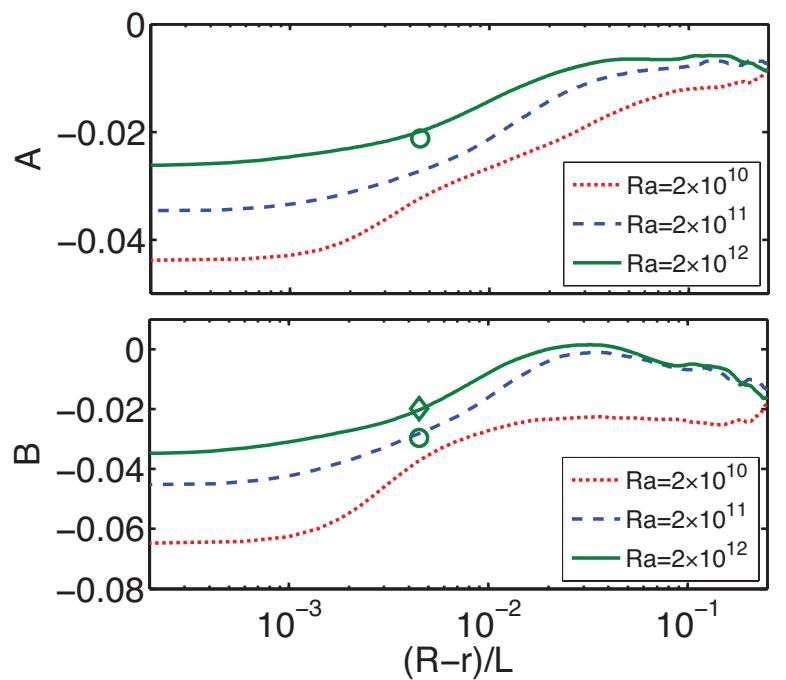

FIG. 4. DNS results for $A$ and $B$ in Eq. 1 for three values of $\mathrm{Ra}$ as a function of $(R-r) / L$ on a logarithmic scale. Coefficients from fits to the averages of $\Theta$ obtained as a function of distance from the bottom and the top plate are shown. The open circles (green online) correspond to the extrapolations of the experimental data for $A$ and $B$ as shown in Figs. 2(a) and (b) to $\mathrm{Ra}=2 \times 10^{12}$. The open diamond indicates the value of $B-\Phi$, see Fig. 2(e) and (b).

They are for the same radial position $(R-r) / L=0.0045$ as that of the experiment, and are based on azimuthally and time averaged temperature data. In this (and the following) figure we show the averages of the profiles determined in the top and the bottom half of the sample. As for the experimental data, the profile at $z / L \gtrsim 10^{-2}$ 
can be described well by Eq. (1). Figure 4 gives the DNS results for $A$ and $B$ as a function of the radial position $(R-r) / L$, based on the temperature data for $10^{-2} \leq z / L \leq 10^{-1}$. In this figure one sees that there is excellent agreement between the values of $A$ and $B$ measured in the experiment and the simulations when $B$ from the experiment is corrected by the offset $\Phi$ at $z / L=0.5$ [see Fig. 2(e)]. In addition, the figure reveals that the magnitude of $A$ is largest near the side wall and that it decreases (approximately logarithmically) as the sample interior is approached. Thus the DNS indicates that the logarithmic vertical temperature profile is strongly influenced by the existence of the side wall.

In this Letter we reported on results obtained by using a combination of experiment and DNS to study the interior of turbulent RBC. For the classical state, which exists for $\mathrm{Ra}<\mathrm{Ra}_{1}^{*}$ and which has laminar BLs adjacent to the top and bottom plates, we find that the bulk which is found between these two layers sustains a non-trivial and interesting temperature field $\Theta(z / L, r / L)$. Whereas it had generally been assumed that the temperature in the sample interior is either constant or varying linearly and slowly in space, we find that $\Theta$ varies logarithmically with distance from the plates over a wide range of $z / L$. The root-mean-square temperature fluctuations show similar variations. The amplitude of the logarithmic profile is largest near the side wall. Its origin remains unclear. On the one hand one may speculate that it is the result of the diffusion of enthalpy carried from the BLs into the interior by plumes; but a model for this process which would yield a logarithmic distribution is not known to us. On the other hand, the logarithmic variation suggests a possible relationship to the well known logarithmic velocity profiles in turbulent shear flows discussed originally by von Kármán [37] and Prandtl [38] (for a recent review, see [39]), and to the recently discovered logarithmic variation of turbulent fluctuations in pipe flow [36]. Perhaps turbulence in the BL may not be a necessary condition for logarithmic profiles - the spatial constraints of the turbulent eddies in the bulk through a boundary layer of Prandtl-Blasius type (with the wall or plate behind it) may also be sufficient for such a logarithmic profile.

In the ultimate state, which exists above $\mathrm{Ra}_{2}^{*}[15,40]$, it was predicted [28] that the BLs are turbulent and that they extend vertically throughout the entire sample; thus there is no "bulk" in the same sense as there is for the classical state. In analogy to turbulent shear flows, a logarithmic temperature profile due to the turbulent BLs was predicted to extend from each plate deep into the sample, with the two profiles meeting at half height. Indeed, the experimental measurements in the ultimate state do find a logarithmic dependence of the temperature on the vertical coordinate. Unfortunately, these large values of $\mathrm{Ra}$ are not yet accessible to DNS (and will not be for some time), and experimental results are available only for one radial position. Thus, the logarithmic variation of the temperature with distance from the plates that was predicted by Grossmann and Lohse [28] has not yet been fully confirmed by simulation or experiment.

It is interesting to note that the parameters $A$ and $B-\Phi$ in Figs. 2(a) and (b) do not show any significant variation over the (unfortunately rather small) accessible range of $\mathrm{Ra}$ in the ultimate state. The corresponding coefficients in shear flow are also independent of the driving [39], which in that case is represented by the Reynolds number Re. Also noteworthy is the fact that the results for $\sigma$ in the ultimate state shown in Fig. 1(b) show no Ra dependence. This, too, mirrors the universal logarithmic dependence found in pipe flow [36]. These comparisons tend to strengthen the likelihood that the logarithmic dependences seen in the ultimate state of RBC are indeed related to those found in shear flows. In distinction to this, in the classical state the coefficients describing $\Theta$ at constant $(R-r) / L$ vary considerably with Ra [see Fig. 1(a) and 2(a) and (b)], suggesting that any relationship to the logarithmic dependences in shear flow, if it exists, is less direct.

Acknowledgements: We are grateful to the Max-Planck Society and the Volkswagen Stiftung for their support of the experiment. We thank the Deutsche Forschungsgemeinschaft (DFG) for financial support through SFB963: "Astrophysical Flow Instabilities and Turbulence". The work of GA was supported in part by the U.S. National Science Foundation through Grant DMR11-58514. The simulation at $R a=2 \times 10^{12}$ was performed as part of a large scale computing project at HLRS (High Performance Computing Center Stuttgart). RJAMS \& DL thank the Foundation for Fundamental Research on Matter (FOM) for financial support.

[1] G. Ahlers, Physics 2, 74 (2009).

[2] G. Ahlers, S. Grossmann, and D. Lohse, Rev. Mod. Phys. 81, 503 (2009).

[3] D. Lohse and K.-Q. Xia, Annu. Rev. Fluid Mech. 42, 335 (2010).

[4] F. Cattaneo, T. Emonet, and N. Weiss, Astrophys. J. 588, 1183 (2003).

[5] F. H. Busse, Chaos 4, 123 (1994).

[6] A. Nordlund, Solar photosphere and convection (Cambridge University press, Cambridge, 2003).

[7] P. Cardin and P. Olson, Phys. of the Earth and Planetary Interiors 82, 235 (1994).

[8] G. Glatzmaier, R. Coe, L. Hongre, and P. Roberts, Nature (London) 401, 885 (1999).

[9] E. van Doorn, B. Dhruva, K. R. Sreenivasan, and V. Cassella, Phys. Fluids 12, 1529 (2000).

[10] D. L. Hartmann, L. A. Moy, and Q. Fu, J. Climate 14, 4495 (2001).

[11] J. Marshall and F. Schott, Rev. Geophys. 37, 1 (1999). 
[12] S. Rahmstorf, Climate Change 46, 247 (2000).

[13] M. Kühn, J. Bosbach, and C. Wagner, Building and Environment 44, 961 (2009).

[14] W. M. Stacey, Fusion: An Introduction to the Physics and Technology of Magnetic Confinement Fusion (Wiley, New York, 2010).

[15] X. He, D. Funfschilling, H. Nobach, E. Bodenschatz, and G. Ahlers, Phys. Rev. Lett. 108, 024502 (2012).

[16] A. Tilgner, A. Belmonte, and A. Libchaber, Phys. Rev. E 47, R2253 (1993).

[17] A. Belmonte, A. Tilgner, and A. Libchaber, Phys. Rev. Lett. 70, 4067 (1993).

[18] A. Belmonte, A. Tilgner, and A. Libchaber, Phys. Rev. E 50, 269 (1994).

[19] Y. B. Xin and K.-Q. Xia, Phys. Rev. E 56, 3010 (1997).

[20] S. L. Lui and K.-Q. Xia, Phys. Rev. E 57, 5494 (1998).

[21] S. Q. Zhou and K.-Q. Xia, Phys. Rev. Lett. 87, 064501 (2001).

[22] J. Wang and K.-Q. Xia, Eur. Phys. J. B 32, 127 (2004).

[23] R. du Puits, C. Resagk, A. Tilgner, F. H. Busse, and A. Thess, J. Fluid Mech. 572, 231 (2007).

[24] Q. Zhou and K.-Q. Xia, Phys. Rev. Lett. 104, 104301 (2010).

[25] R. J. A. M. Stevens, Q. Zhou, S. Grossmann, R. Verzicco, K.-Q. Xia, and D. Lohse, Phys. Rev. E 85, 027301 (2012).

[26] E. Brown and G. Ahlers, Europhys. Lett. 80, 14001 (2007).

[27] At a more detailed level it has long been recognized that the bulk actually sustains small temperature gradients, but these gradients were believed to be more or less in- dependent of vertical position and the total temperature drop across the bulk is known to be much smaller than that across the BLs (see, for instance, $[16,26]$ ).

[28] S. Grossmann and D. Lohse, Phys. Fluids 23, 045108 (2011).

[29] G. Ahlers, D. Funfschilling, and E. Bodenschatz, New J. Phys. 11, 123001 (2009).

[30] G. Ahlers, X. He, D. Funfschilling, and E. Bodenschatz, (arXiv:physics.flu-dyn/1205.0108) (2012).

[31] R. J. A. M. Stevens, R. Verzicco, and D. Lohse, J. Fluid Mech. 643, 495 (2010).

[32] R. J. A. M. Stevens, D. Lohse, and R. Verzicco, J. Fluid Mech. 688, 31 (2011).

[33] G. Ahlers, E. Brown, and A. Nikolaenko, J. Fluid Mech. 557, 347 (2006).

[34] E. Brown and G. Ahlers, Phys. Fluids 20, 105105 (2008).

[35] E. Brown, A. Nikolaenko, and G. Ahlers, Phys. Rev. Lett. 95, 084503 (2005).

[36] M. Hultmark, M. Vallikivi, S. Bailey, and A. Smits, Phys. Rev. Lett 108, 094501 (2012).

[37] T. von Kármán, Nachr. Ges. Wiss. Göttingen, Math.Phys. Kl. 58-76, 322 (1930).

[38] L. Prandtl, Ergeb. Aerodyn. Versuch, Göttingen IV, 18 (1932).

[39] I. Marusic, B. J. McKeon, P. A. Monkewitz, H. M. Nagib, A. J. Smits, and K. R. Sreenivasan, Phys. Fluids 22, 065103 (2010).

[40] S. Grossmann and D. Lohse, Phys. Rev. E 66, 016305 (2002). 